Somnologie $2018 \cdot 22: 77-78$

https://doi.org/10.1007/s11818-018-0160-x

(c) Springer Medizin Verlag GmbH, ein Teil von Springer Nature 2018

CrossMark

Boris A. Stuck

Klinik für Hals,-Nasen- und Ohrenheilkunde, Universitätsklinikum Gießen und Marburg GmbH, Marburg Deutschland

\title{
CPAP-Alternativen bei der obstruktiven Schlafapnoe
}

rüber, wie denn nun eine ausreichende Compliance definiert werden soll und wie groß diese im Alltag wirklich ist (die Studienlage hierzu ist alles andere als eindeutig), stand hierbei einer nüchternen Betrachtung des grundsätzlichen Problems häufig im Wege.

Aber auch in Bezug auf den therapeutischen Nutzen der CPAP-Therapie haben in den letzten Jahren neuere Studien gewisse Zweifel gesät. Gerade die Daten der „Save“-Studie, die an einem großen Kollektiv von Patienten mit OSA ohne schwere Tagesschläfrigkeit keinen Vorteil der CPAP-Therapie in Bezug auf das kardiovaskuläre Überleben gezeigt hat, lässt fraglich erscheinen, ob Patienten ohne relevante Tagessymptomatik überhaupt von der Therapie profitieren, wenn man statt kurzfristiger Surrogat-Parameter das kardiovaskuläre Überleben betrachtet. Die isolierte Fokussierung auf die CPAP-Therapie weicht daher zunehmend einer differenzierten Betrachtung, die sich fortbewegt von einer „one treatment fits all“-Strategie zu einer individualisierten Therapie der OSA.

Auch wenn eine Reihe therapeutischer Alternativen schon lange verfügbar ist, so haben diese teils erst in den letzten Jahren breitere Akzeptanz gefunden. Die Studienlage zu vielen dieser Therapien ist noch immer weit entfernt von der verfügbaren Literatur zur CPAP-Therapie, was sowohl die Anzahl der Studien als auch die methodische Qualität der Arbeiten anbelangt. Doch auch hier beginnen die therapeutischen Alternativen zunehmend wissenschaftlich an Boden gut zu machen.

Die breiteste Akzeptanz haben sicher die Unterkieferprotrusionsschienen gefunden, die zumindest für die leicht- bis mittelgradige OSA als gleichwertige Alternative zur CPAP-Therapie anerkannt sind, sofern die allgemeinen und anatomischen Voraussetzungen für eine solche Therapie (ausreichender Unterkiefervorschub und Zahnstatus, limitierte Adipositas, etc.) gegeben sind. Zu dieser Therapieform wurde in den letzten Jahren viel publiziert, insbesondere auch in der Somnologie.

Die vorliegende Ausgabe bezieht sich daher explizit auf die weiteren, weniger gut etablierten Alternativen. Vonk und ihre Mitautorin beleuchten in diesem Zusammenhang die Möglichkeiten der Rückenlageverhinderung bei Patienten mit lageabhängiger OSA, insbesondere unter Berücksichtigung der neu verfügbaren Hilfsmittel, und unterstreichen dabei die Bedeutung des Phänomens der Lageabhängigkeit. Zwei Übersichten befassen sich mit operativen Verfahren. Während $\mathrm{zu}$ einem der ältesten Therapieverfahren - der Tonsillektomie mit Uvulopalatopharyngoplastik (TEUPPP) - eine Kurzfassung einer umfassenden systematischen Übersichtsarbeit präsentiert wird, beschreiben Hofauer et al. die aktuelle Situation zum Einsatz der Stimulationstherapie des oberen Atemwegs in Deutschland. Die Arbeit von Schmid et al. bezieht sich auf die konservative Therapie der Adipositas als des wichtigsten ursächlichen Risikofaktors für die Schlafapnoe. Der Beitrag zur Schlafvideoendoskopie von Edenharter et al. erläutert die Grundlagen eines zunehmend verbreiteten Untersuchungsverfahrens zur Differenzierung der Atemwegsobstruktionen, während Wessolleck et al. den Einsatz der Muskelstimulation beim primären Schnarchen betrachten. Eine Falldarstellung zur tional geführte Auseinandersetzung da- 


\section{Editorial}

interdisziplinären Diagnostik und alternativen Therapie der OSA rundet die Zusammenstellung ab.

Entscheidend für den Therapieerfolg wird zukünftig sein, gemeinsam mit dem Patienten eine individualisierte Therapiestrategie für seine Erkrankung zu entwickeln. Die Versuche, die OSA weiter zu phänotypisieren, wird diese Entwicklung noch beschleunigen. Während die CPAPTherapie, von der individuellen Patientenpräferenz abgesehen, für nahezu alle Fälle geeignet erscheint, so gestaltet sich die Indikationsstellung für die genannten therapeutischen Alternativen anspruchsvoll und erfordert umfassende Kenntnisse zu den therapeutischen Möglichkeiten. Die Autoren hoffen, dass diese Ausgabe der Somnologie hierzu ihren Beitrag leistet.

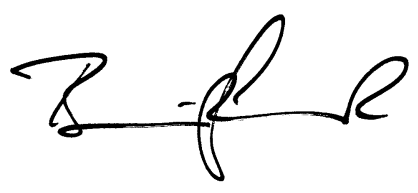

Prof. Dr. med. Boris A. Stuck

\section{Korrespondenzadresse}

Prof. Dr. med. B. A. Stuck
Klinik für Hals,-Nasen-
und Ohrenheilkunde,
Universitätsklinikum Gießen
und Marburg GmbH
Baldingerstraße, 35043 Mar-
burg, Deutschland
boris.stuck@uk-gm.de

Interessenkonflikt. B.A. Stuck gibt an, dass kein Interessenkonflikt besteht. 